\title{
Ambipolar charge transport in organic field-effect transistors
}

\author{
Edsger C. P. Smits, ${ }^{1,2,3, *}$ Thomas D. Anthopoulos, ${ }^{4}$ Sepas Setayesh, ${ }^{2}$ Erik van Veenendaal,,${ }^{5}$ Reinder Coehoorn, ${ }^{2}$ \\ Paul W. M. Blom, ${ }^{1}$ Bert de Boer, ${ }^{1}$ and Dago M. de Leeuw ${ }^{2}$ \\ ${ }^{1}$ Molecular Electronics, Material Science Centre, University of Groningen, Nijenborgh 4, 9747 AG Groningen, The Netherlands \\ ${ }^{2}$ Philips Research Laboratories High Tech Campus 4 (WAG 11) 5656 AE Eindhoven, The Netherlands \\ ${ }^{3}$ Dutch Polymer Institute, Nijenborgh 4, 9747 AG Groningen, The Netherlands \\ ${ }^{4}$ Department of Physics, Blackett Laboratory, Imperial College London, London SW7 2BW, United Kingdom \\ ${ }^{5}$ Polymer Vision, Philips Technology Incubator, High Tech Campus 48, 5656 AE Eindhoven, The Netherlands
}

(Received 26 January 2006; published 9 May 2006)

\begin{abstract}
A model describing charge transport in disordered ambipolar organic field-effect transistors is presented. The basis of this model is the variable-range hopping in an exponential density of states developed for disordered unipolar organic transistors. We show that the model can be used to calculate all regimes in unipolar as well as ambipolar organic transistors, by applying it to experimental data obtained from ambipolar organic transistors based on a narrow-gap organic molecule. The threshold voltage was determined independently from metal insulator semiconductor diode measurements. An excellent agreement between theory and experiment is observed over a wide range of biasing regimes and temperatures.
\end{abstract}

DOI: 10.1103/PhysRevB.73.205316 PACS number(s): 73.50.-h, 73.61.Ph, 73.43.Cd, 73.40.Qv

\section{INTRODUCTION}

Organic thin-film field-effect transistors (FETs) have been studied extensively over the past decade, and tremendous progress has been achieved in device performance ${ }^{1,2}$ Organic FETs have generally been used as unipolar devices, and this has limited the design of integrated circuits to unipolar logic. From a performance point of view, complementary logic is crucial. The advantages are low-power dissipation, good noise margin and robust operation.

The first organic complementary logic circuits, 48-stage shift registers, were created by combining discrete $p$-channel and $n$-channel transistors. ${ }^{3,4}$ Two different semiconductors were used, one for the $p$-channel and one for the $n$-channel. Both materials had to be deposited and patterned locally and sequentially. With such an approach it is difficult to minimize the parameter spread and match the $n$ - and $p$-channel transconductances. A step forward for the development of complimentary logic is the realization of ambipolar transistors based on a single semiconducting film and a single type of electrode.

Numerous groups have now demonstrated ambipolar transistors,${ }^{5-10}$ discrete inverters, ${ }^{11-13}$ and simple circuits. ${ }^{14}$ An analytical expression for the charge transport that can be incorporated in circuit simulators is required for the design of complementary-like logic. Here we present a model that can be used to describe current transport in unipolar as well as ambipolar transistors. The model predictions are tested against experimental data obtained from ambipolar organic transistors based on a narrow-gap organic molecule.

\section{EXPERIMENTAL}

Field-effect transistors were fabricated on a doped $\left(n^{++}\right)$ silicon wafer acting as the gate electrode with a $200-\mathrm{nm}-$ thick layer of thermally grown silicon dioxide as gate dielectric. The surface of the $\mathrm{SiO}_{2}$ insulator was subsequently passivated by a hexamethyldisilazane (HMDS) treatment. Gold source and drain contacts were defined using standard photolithographic techniques. Titanium was used as an adhesion layer. To minimize in-plane parasitic leakage currents, a circular device geometry was used. ${ }^{15}$ The channel width of the ring transistors was $1000 \mu \mathrm{m}$ and the channel length varied from 2.5 to $40 \mu \mathrm{m}$. Electrical characterization of the transistors was performed using an Agilent 4155C Semiconductor Parameter Analyzer under vacuum $\left(10^{-7} \mathrm{mbar}\right)$ and in dark.

Metal insulator semiconductor (MIS) diodes were made starting from the same oxidized silicon wafer as used for the transistor test devices. The semiconductor was spin-coated and subsequently $50 \mathrm{~nm}$ of gold was evaporated through a shadow mask as the top electrode. The area of the top electrode was varied from 0.07 to $0.38 \mathrm{~cm}^{2}$. The impedance measurements were performed with an Schlumberger $1260 \mathrm{Im}$ pedance Gain-Phase Analyzer under vacuum $\left(10^{-7} \mathrm{mbar}\right)$ and in dark. ${ }^{16}$

As an ambipolar semiconductor, a small band-gap organic molecule, nickel-dithiolene (NiDT) [see Fig. 1(a)], was used. This material was obtained from Sensient $\mathrm{GmbH}$, Germany. Cyclic voltammetry measurements in solution with $\mathrm{Ag} / \mathrm{Ag}^{+}$ as the reference electrode in acetonitrile reveal a narrow energy gap of $0.9 \mathrm{eV}$ with the highest occupied molecular orbital (HOMO) and lowest unoccupied molecular orbital (LUMO) levels at 5.2 and $4.3 \mathrm{eV}$, respectively [see Fig. 1(b)]. This value is in good agreement with the optical band gap obtained from the solid-state absorption spectrum, where a maximum absorption around a wavelength of $1160 \mathrm{~nm}$ with the onset at around $1450 \mathrm{~nm}$ is observed. The Fermi level of gold (approximately $4.8 \mathrm{eV}$ ) is in between the HOMO and LUMO energy levels of NiDT. This implies that the barrier for injection of holes in the HOMO and for electrons in the LUMO is expected to be smaller than $0.5 \mathrm{eV}$. These small injection barriers explain the occurrence of ambipolar transport in the field-effect transistors.

Nickel dithiolene was found to be highly soluble in a range of chlorinated organic solvents. Thin films were spun from a solution of $5 \mathrm{mg} / \mathrm{mL}$ of NiDT in chloroform at 
a)

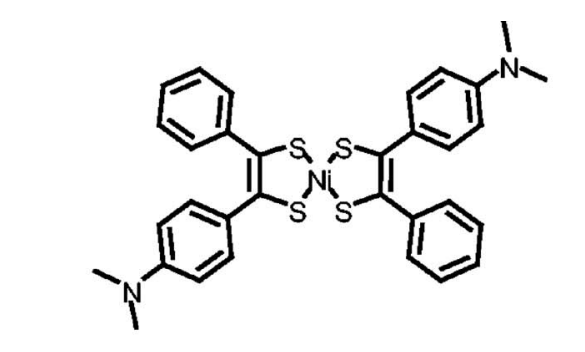

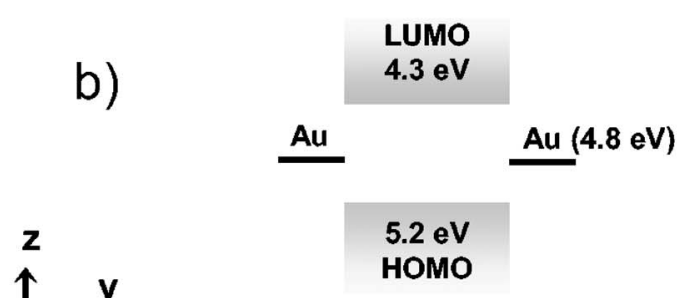

c)

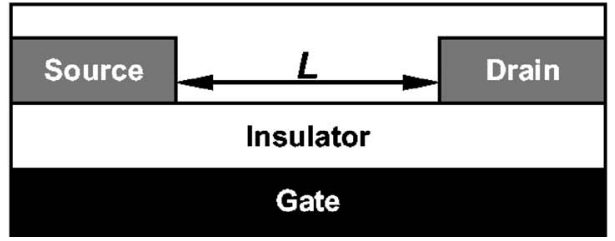

НОМо

Top electrode (Ground)

d)

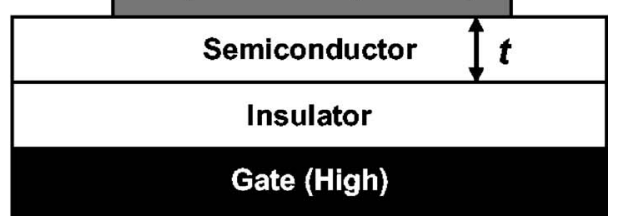

FIG. 1. (a) Molecular structure of nickel dithiolene (bis[4-dimethylaminodithiobenzyl]-nickel). (b) Band diagam of nickel dithiolene. (c) Schematics of a bottom gate and contact transistor similar to the one used for the experiments. The $x, y$, and $z$ directions are indicated to visualize the directions referred to in the text. (d) Schematic representation of a MIS diode used in the experiments.

$500 \mathrm{rpm}$. The devices were then annealed at $60{ }^{\circ} \mathrm{C}$ for $1 \mathrm{~h}$ under vacuum.

The transistors function in air and light. When stored in ambient conditions, no sign of electrical degradation is observed for the hole as well as the electron current for days. However, the hysteresis between backward and forward scans increases upon exposure to air. Therefore, electrical characterization of both transistors and MIS diodes was performed in dark and vacuum.

\section{CHARGE TRANSPORT MODELING}

Due to disorder and variation of interaction energies, thin films of disordered semiconductors do not have two delocalized energy HOMO and LUMO bands separated by an energy gap. Instead, a spatial and energetic spread of charge transport sites will be present, often approximated in shape by a Gaussian density of states (DOS). ${ }^{17}$ The use of that approximation is supported by the observation of Gaussianshaped absorption spectra of disordered polymers. Furthermore, for disordered small-molecule systems, the electrostatic field from a random distribution of static or induced dipoles leads to a Gaussian DOS function. ${ }^{18}$ For a system with both negligible background doping and at typical gateinduced carrier densities, the carrier mobility resulting from hopping in a Gaussian DOS can be approximated by the mobility resulting from hopping in an exponential DOS. ${ }^{19-21}$ The often observed almost linear relationship (on a double$\log$ scale) between the field-effect mobility and the carrier concentration at the gate-dielectric/semiconductor interface ${ }^{21}$ suggests that an exponential DOS is nevertheless a good approximation for the DOS in the case of intermediate and high carrier concentrations. ${ }^{19}$ Therefore, our analyses of the $I(V)$ characteristics of unipolar and ambipolar organic transistors will be based on the mobility in an exponential DOS. In the literature, such a dependency was also found for amorphous silicon. $^{22,23}$
Electrical transport is described by hopping between the localized states. With increasing carrier density, the filling of tail states of the DOS increases. The charge carriers have more transport states available at higher energy and thus also the average mobility increases. For bulk conduction, a transport model has been derived based on variable range hopping and percolation. ${ }^{19}$ This model gives an analytical description for the bulk conductivity as a function of carrier density. In an organic field-effect transistor (OFET) in the linear regime, where $V_{d} \rightarrow 0$, the potential in the channel is to a good approximation uniform between source and drain. The model combined with an exponential DOS was used to calculate the current as a function of gate bias. For the linear regime, an accurate description of the transport as a function of the temperature and gate biasing has been demonstrated. ${ }^{19-21,24}$ Here, we show that the formalism can be applied to a wide range of bias conditions. This will be shown for both unipolar and ambipolar OFETs.

A schematic layout of a field-effect transistor is presented in Fig. 1(c), where $L$ is the channel length, $W$ is the channel width in the $y$ direction and $t$ is the semiconductor thickness. The $x$ direction is the direction between source and drain and the $z$ direction is the direction perpendicular to the channel. For the derivation of the current voltage $I(V)$ characteristics, we ignored the source and drain contact resistances. Furthermore, we consider long channels so that short channel effects can be disregarded and contact resistance effects are minimalized. Finally, the gradual channel approximation is used. The electric field in the $z$ direction perpendicular to the film is much larger than in the parallel $x$ direction. In this way, the transport can be treated independently in both directions. First, we calculate the sheet conductance as a function of local effective gate potential in the channel. The chargecarrier density in the sheet as a function of effective gate potential and position $z$ can be solved analytically for an exponential DOS. We substitute the relation between bulk conductivity and charge-carrier density. The sheet conductance then follows from integration over the charge-carrier 
density profile. The source-drain current as a function of gate bias is then obtained by integrating the sheet conductances along the channel.

\section{A. Unipolar transport}

The drain bias, $V_{d}$, gives rise to a nonzero value of the local electrochemical potential at each point in the channel, $V_{x}$. The difference between this electrochemical potential and the gate bias, $V_{g}$, is the effective gate potential, $V_{\text {eff }}$. This effective potential determines the amount of induced charge at position $x$. The effective potential is given by

$$
V_{\text {eff }}=V_{g}-V_{t}-V_{x},
$$

where $V_{t}$ is the threshold voltage. ${ }^{20}$ The correction for the threshold voltage is included to account for the presence of trapped and static charges. To be specific, we focus here on electron transport so that $V_{\text {eff }}>0$ in order to have accumulation. At each point $x$, the charge-carrier density decreases with increasing distance from the insulator/semiconductor interface, $z$. This charge distribution depends on the effective gate potential. For an exponential DOS, this charge distribution, $n(z)$, has been calculated from the Poisson equation ${ }^{26}$ and is given by

$$
n(z)=\frac{2 k_{B} T_{0} \varepsilon_{s} \varepsilon_{0}}{q^{2}\left(z+z_{0}\right)^{2}}
$$

with

$$
z_{0}=\frac{2 k_{B} T_{0} \varepsilon_{s} \varepsilon_{0}}{q C_{i} V_{\mathrm{eff}}},
$$

where $k_{B} T_{0}$ is the width of the exponential DOS, $k_{B}$ is the Boltzmann constant, $\varepsilon_{0}$ is the relative permittivity of vacuum, $\varepsilon_{s}$ is the dielectric constant of the semiconductor, $C_{i}$ is the gate capacitance per unit area, and $q$ is the electron charge. Equations (2) and (3) show that the carrier density decreases with distance from the interface. Calculations of the effective accumulation layer thickness, $z_{0}$, show it is typically at most a few nanometers.

From percolation theory, the following expression for the conductivity has been derived as a function of the carrier density and temperature: ${ }^{19}$

$$
\sigma(n)=\sigma_{0}\left(\frac{\left(\frac{T_{0}}{T}\right)^{4} \sin \left(\pi \frac{T}{T_{0}}\right)}{(2 \alpha)^{3} B_{c}}\right)^{T_{0} / T} n^{T_{0} / T},
$$

where $\sigma_{0}$ is a conductivity prefactor, $\alpha^{-1}$ is the wave function overlap localization length, $B_{c}$ is the critical number for the onset of percolation ( $\sim 2.8$ for 3D amorphous systems), and $T$ is the temperature. A superlinear increase in conductivity is observed for an exponential DOS with charge density. ${ }^{19}$ This expression holds for disordered semiconductors where transport occurs solely through localized states and at temperatures $T$ well below $T_{0}$.

For each value of the local effective potential, $V_{\text {eff }}$, substitution of Eqs. (2) and (3) in Eq. (4) gives the conductivity, $\sigma(z)$, as a function of the distance $z$ from the interface. Inte- grating this conductivity, $\sigma(z)$, over the layer thickness then yields the sheet conductance, $G_{\mathrm{sh}}\left(V_{\mathrm{eff}}\right)$,

$$
G_{\mathrm{sh}}\left(V_{\mathrm{eff}}\right)=\int_{0}^{t} \sigma(z) d z \cong \frac{T}{2 T_{0}-T} q \mu_{\mathrm{FE}}\left(V_{\mathrm{eff}}\right) C_{i} V_{\mathrm{eff}}
$$

with

$$
\left.\mu_{\mathrm{FE}}\left(V_{\mathrm{eff}}\right)=\frac{\sigma_{0}}{q}\left(\frac{\left(\frac{T_{0}}{T}\right)^{4} \sin \left(\pi \frac{T}{T_{0}}\right)}{(2 \alpha)^{3} B_{c}}\right)\right)^{T_{0} / T}\left(\frac{\left(C_{i} V_{\mathrm{eff}}\right)^{2}}{2 k_{b} T_{0} \varepsilon_{S} \varepsilon_{0}}\right)^{\left(T_{0} / T\right)-1},
$$

where $\mu_{\mathrm{FE}}\left(V_{\mathrm{eff}}\right)$ is the local field-effect mobility. ${ }^{19}$ The righthand part of Eq. (5) has been obtained assuming that the carrier density is negligible at the top of the semiconductor film, so that effectively $t=\infty$. The source-drain current $I_{\text {sd }}$, which is constant within the channel (independent of the position $x$ ), is given by

$$
I_{\mathrm{sd}}=-W G_{\mathrm{sh}}\left(V_{\mathrm{eff}}\right) \frac{\partial V_{\mathrm{eff}}(x)}{\partial x} .
$$

Integrating over the length of the transistor and replacing $V_{\text {eff }}$ by $V_{g}-V_{t}-V_{x}$ gives

$$
I_{\mathrm{sd}}=-\frac{W}{L} \int_{0}^{V_{\mathrm{d}}} G_{\mathrm{sh}}\left(V_{g}-V_{t}-V_{x}\right) d V_{x} .
$$

The source-drain current then follows immediately by integrating the sheet conductance, Eq. (5), over the electrochemical potential between the source and drain bias, which yields

$$
I_{\mathrm{sd}}=\gamma \frac{W}{L} \frac{T}{2 T_{0}} \frac{T}{2 T_{0}-T}\left[\left(V_{g}-V_{t}\right)^{2 T_{0} / T}-\left(V_{g}-V_{t}-V_{d}\right)^{2 T_{0} / T}\right]
$$

for $V_{g}-V_{t} \geq V_{d}$ with

$$
\gamma=\frac{\sigma_{0}}{q}\left(\frac{\left(\frac{T_{0}}{T}\right)^{4} \sin \left(\pi \frac{T}{T_{0}}\right)}{(2 \alpha)^{3} B_{c}}\right)^{T_{0} / T}\left(\frac{1}{2 k_{b} T_{0} \varepsilon_{S} \varepsilon_{0}}\right)^{\left(T_{0} / T\right)-1} C_{i}^{\left(2 T_{0} / T\right)-1} .
$$

We note that Eq. (9) holds for $V_{g}-V_{t}>V_{d}$. When $V_{g}-V_{t}$ $<V_{d}$, the transistor is operated in saturation. When $V_{d}$ is exactly equal to $V_{g}-V_{t}$, the effective potential at the drain contact is zero, $V_{\text {eff }}=0$. Hence at the drain contact there is no accumulation of charge carriers. In general, the channel is pinched off when $V_{\text {eff }}$ is zero. This occurs when the electrochemical potential is equal to the applied gate bias. With increasing drain bias, the position at which the pinchoff occurs moves into the channel from the drain in the direction of the source contact. When pinchoff occur, the accumulation length is smaller than the channel length. We ignore this channel shortening in the equations. Moreover, as is known from the literature, the resistance of the depleted part of the channel can be disregarded. ${ }^{27}$ With these assumptions, the source-drain current in saturation is derived from Eq. (8) by 
replacing $V_{d}$ with $V_{g}-V_{t}$. The saturated source-drain current is given by

$$
I_{\mathrm{sd}}^{\mathrm{sat}}=I_{\mathrm{sd}}\left(V_{d}=V_{g}-V_{t}\right)=\gamma \frac{W}{L} \frac{T}{2 T_{0}} \frac{T}{2 T_{0}-T}\left(V_{g}-V_{t}\right)^{2 T_{0} / T}
$$

for $V_{g}-V_{t}<V_{d}$. A more general expression for the sourcedrain current has been derived by Calvetti et al. ${ }^{25}$ The derivation included the diffusion current and the effect of a finite semiconductor thickness, $t$. However, we find that our more simplified approach provides a fully adequate description for the transport regimes investigated in this paper. The derivation presented here yields the analytical expression Eq. (8), which will be crucial for deriving the ambipolar transport model further on.

In the limit of a negligible drain bias $\left(V_{d}\right), I_{\mathrm{sd}}$ is a linear function of $V_{d}$, Eq. (8), and is consistent with results for this regime given in the literature. ${ }^{19,20}$ In the derivation, we used a gate bias-dependent mobility. For most inorganic devices and some organic single crystals, the mobility is constant as a function of gate bias. ${ }^{28}$ This corresponds to taking $T=T_{0}$. The classical metal oxide semiconductor field-effect transistor (MOSFET) equations are then obtained, ${ }^{29}$ although the mobility obtained from the percolation theory, Eq. (6), is not expected to hold for this limit. The classical MOSFET equations have been used for modeling of (ambipolar) OFETs. ${ }^{27,30}$ The compact modeling given by Eqs. (9)-(11) is valid for $T<T_{0}$. Several authors have proposed that for empirically modeling OFETs, the same functional dependence can be applied,

$$
I_{\mathrm{sd}}=a\left[\left(V_{g}-V_{t}\right)^{b}-\left(V_{g}-V_{t}-V_{d}\right)^{b}\right]
$$

where $a$ and $b$ are fit constants. ${ }^{31,32}$ Other authors have used numerical simulations based on (ambipolar) amorphous silicon theory ${ }^{33}$ to model OFETs. ${ }^{34}$

\section{B. Ambipolar transport}

Depending on the bias conditions, the current in an ambipolar transistor is due to electrons, holes, or both. The operating regimes are schematically presented in Fig. 2. For each regime, the charge distribution in the channel and the output curves are schematically given. When $\left|V_{g}-V_{t}\right|>\left|V_{d}\right|$ and both are positive, the current is carried by electrons (regime 1). The current as a function of bias voltages is then given by Eq. (9) as derived in the preceding section for a unipolar electron-only transistor. Similarly, when $\left|V_{g}-V_{t}\right|>\left|V_{d}\right|$ and both are negative, the current is carried by holes (regime 4). The hole current as a function of bias voltage is given by the same expression by replacing $V_{g}-V_{t}$ by $-V_{g}+V_{t}, V_{d}$ by $-V_{d}$, and taking the source drain current to be negative. We note that electrons transport through the unoccupied density of states corresponding to the LUMO levels while the holes transport through the occupied density of states corresponding to the HOMO levels. This implies that for holes and electrons, the parameters $T_{0}, \sigma_{0}$, and $\alpha^{-1}$ are different. When $V_{g}-V_{t}$ is positive and $V_{d}$ theis negative (regime 6), the effective gate potential is positive throughout the whole chan-

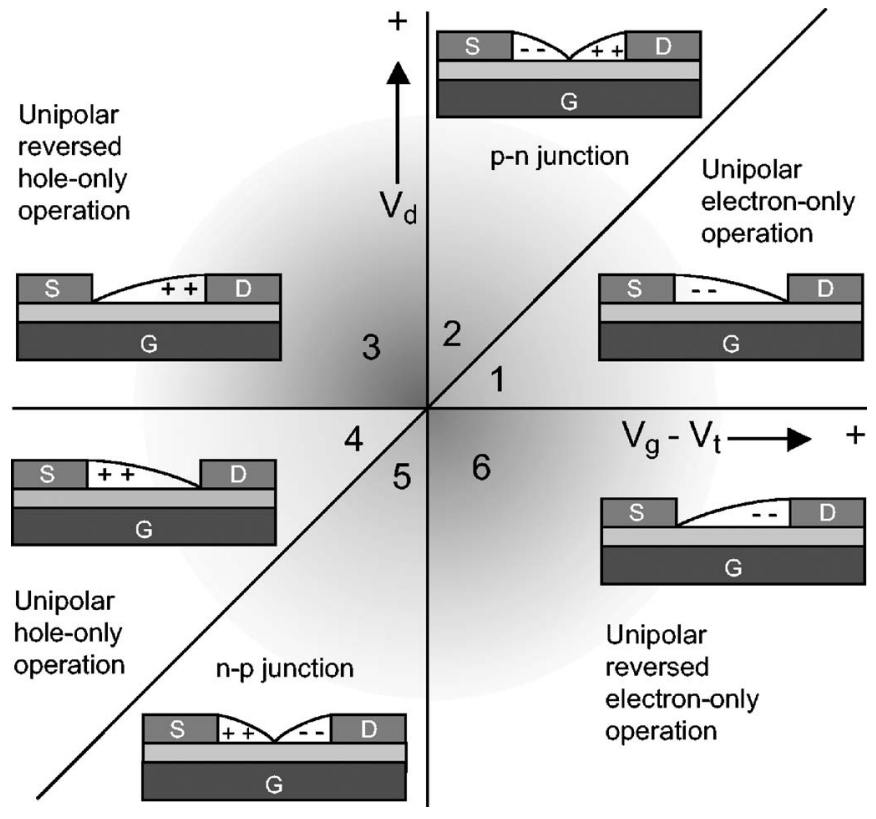

FIG. 2. A sketch of all operating regimes for a typical ambipolar transistor as a function of drain and gate biasing.

nel. The transistor operates as unipolar $n$-type transistor where the source and drain are inverted. Therefore, the current in regime 6 is given by Eq. (9) in which $V_{g}$ is replaced by $V_{g}-V_{d}$ and $V_{d}$ is replaced by $-V_{d}$, which gives Eq. (9) again

$$
\begin{aligned}
I_{\mathrm{sd}}= & \frac{W}{L} \gamma_{e} \frac{T}{2 T_{0, e}} \frac{T}{2 T_{0, e}-T}\left[\left(V_{g}-V_{t}\right)^{2 T_{0, e} / T}\right. \\
& \left.-\left(V_{g}-V_{t}-V_{d}\right)^{2 T_{0, e} / T}\right]
\end{aligned}
$$

for $V_{g}-V_{t}<0$.

Similarly, when $V_{g}-V_{t}$ is negative and $V_{d}$ is positive (regime 3), the effective gate potential throughout the channel is negative. The hole current is given by Eq. (13) upon applying the appropriate substitutions. Ambipolarity can occur when $V_{d}>V_{g}-V_{t}>0$ (regime 2) and when $V_{d}<V_{g}-V_{t}<0$ (regime 5). Under those bias conditions, a unipolar transistor then operates in saturation. The channel is pinched off and charges cannot be accumulated in the pinched off part of the channel. In an ambipolar transistor, those bias conditions give rise to a change of the sign of the effective gate potential at a certain position in the channel. Holes and electrons are accumulated at opposite sides. A narrow transition region, which acts as a $p n$-junction, separates the accumulation regions. We now develop a description of the charge transport in this regime.

For simplicity, we assume bimolecular recombination of electrons and holes with an infinite rate constant, and a constant threshold voltage throughout the entire channel, i.e., equal threshold voltages in the electron and hole accumulation region. For infinite recombination, electrons and holes cannot pass each other without recombining. Therefore, recombination takes place at a single plane, $x=x_{0}$, namely the plane where the effective gate potential is zero. The ambipolar transistor is then represented by a unipolar $n$-type transis- 
tor in series with a unipolar $p$-type transistor, with channel lengths $x_{0}$ and $L-x_{0}$, respectively. The injected electron current (regime 1) is

$$
I_{\text {sd }, e}\left(x<x_{0}\right)=\frac{W}{x_{0}}\left(\gamma_{e} \frac{T}{2 T_{0, e}} \frac{T}{2 T_{0, e}-T}\left(V_{g}-V_{t}\right)^{2 T_{0, e} / T}\right)
$$

and the injected hole current is

$$
\begin{aligned}
I_{\mathrm{sd}, h}\left(x_{0}<x<L\right)= & \frac{W}{L-x_{0}}\left(\gamma_{h} \frac{T}{2 T_{0, h}} \frac{T}{2 T_{0, h}-T}\right. \\
& \left.\times\left(-V_{g}+V_{t}+V_{d}\right)^{2 T_{0, h} T}\right) .
\end{aligned}
$$

We apply the condition that both currents are equal because recombination is infinite. This results in spatial separation of the electron and hole area and we can then derive, $x_{0}$, the position of the $p n$-junction,

$$
x_{0}=\frac{\left(V_{g}-V_{t}\right)^{\left(2 T_{0, e} / T\right)-1}}{\left(V_{g}-V_{t}\right)^{\left(2 T_{0, e} / T\right)-1}+C\left(V_{d}-V_{g}-V_{t}\right)^{\left(2 T_{0, h} / T\right)-1}} L
$$

with

$$
C=\frac{\left(2 T_{0, e}-T\right)}{\left(2 T_{0, h}-T\right)} \frac{2 T_{0, e}}{2 T_{0, h}} \frac{\gamma_{h}}{\gamma_{e}} .
$$

Equation (16) shows how the plane at which recombination takes place shifts from $x_{0}=0$ when $V_{\text {eff }}=0$ to $x_{0}=L$ when $V_{\text {eff }}=V_{d}$. Substitution of the position of the expression for $x_{0}$ in Eq. (14) or Eq. (15) yields an expression for the current in an ambipolar field-effect transistor from which the $p n$-junction position $x_{0}$ is eliminated,

$$
\begin{aligned}
I_{\mathrm{sd}}= & \frac{W}{L}\left(\gamma_{e} \frac{T}{2 T_{0, e}} \frac{T}{2 T_{0, e}-T}\left(V_{g}-V_{t}\right)^{2 T_{0, e} / T}+\gamma_{h} \frac{T}{2 T_{0, h}} \frac{T}{2 T_{0, h}-T}\right. \\
& \left.\times\left(-V_{g}+V_{t}+V_{d}\right)^{2 T_{0, e} / T}\right) .
\end{aligned}
$$

A similar expression can be derived for regime 5. From fits to the experimental current, we obtain all parameters needed to calculate the position of the $p n$-junction.

Surprisingly, the current that is given by Eq. (18) is exactly equal to the sum of the electron and hole currents that would be present in the absence of a current of the other polarity: the ambipolar transistor is then represented by a unipolar $n$-type transistor in parallel with a unipolar $p$-type transistor, both operating in saturation [Eq. (11)]. The hole and electron currents are constant, but not necessarily equal, throughout the whole channel. We regard the assumption that the electrons and holes move independently, and that there is no $p n$-junction, as unphysical. We note that we cannot determine the recombination rate from the experimental currentvoltage characteristics with the current model. However, measurements of the position and the width of the $p n$-junction should allow probing of the recombination rate.
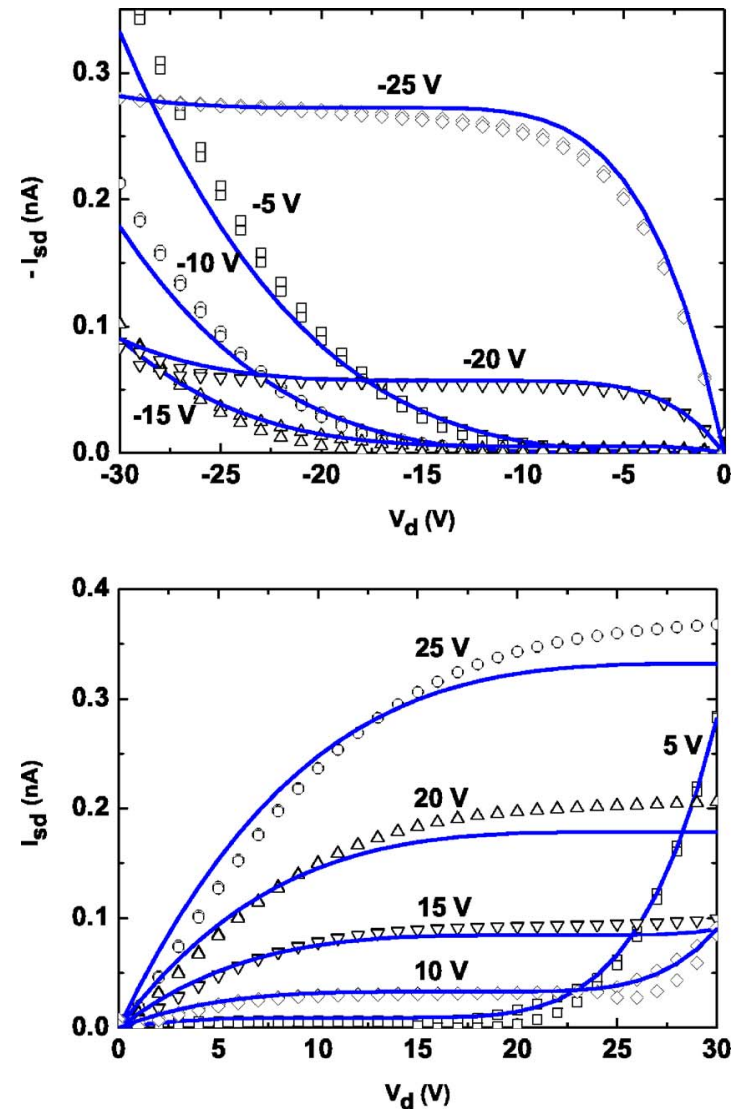

FIG. 3. (Color online) Output characteristics for negative (a) and positive (b) gate biases. The output characteristics presented were measured at $253 \mathrm{~K}$ for a channel length of $20 \mu \mathrm{m}$. The symbols represent the data points and the solid lines represent the theoretical fit.

\section{AMBIPOLAR NICKEL DITHIOLENE FIELD-EFFECT TRANSISTORS}

As a model compound to analyze charge transport in an ambipolar field-effect transistor, we used films based on the small band-gap molecule NiDT as the semiconductor. The injection barriers for both holes and electrons are expected to be less then $0.5 \mathrm{eV}$. These barriers are small enough to allow ambipolar charge injection. No special treatment of the gate dielectric was performed to prevent electron trapping. ${ }^{35}$ These transistors function in air and light; however, to minimize charge trapping by water or oxygen, all measurements were performed in vacuum.

The transistors were characterized by output $\left[I_{\mathrm{sd}}\left(V_{d}\right)\right]$ and transfer $\left[I_{\mathrm{sd}}\left(V_{g}\right)\right]$ curves measured as a function of temperature. Typical output curves measured at $253 \mathrm{~K}$ are presented in Fig. 3. The drain bias $\left(V_{d}\right)$ was swept back and forth. No hysteresis is observed. In Fig. 3(a), both a negative drain and gate bias were used. This corresponds to regimes 4 and 5 in Fig. 2. Positive biases were used in Fig. 3(b). This corresponds to regimes 1 and 2 in Fig. 2. Symbols represent actual data points and the solid lines are theoretical fits to the data according to Eqs. (9) and (18). The output curves of Fig. 3 show at low gate bias and high drain biases a pronounced increase of current with $V_{d}$. This is typical for an ambipolar transistor and not present in a unipolar one. 

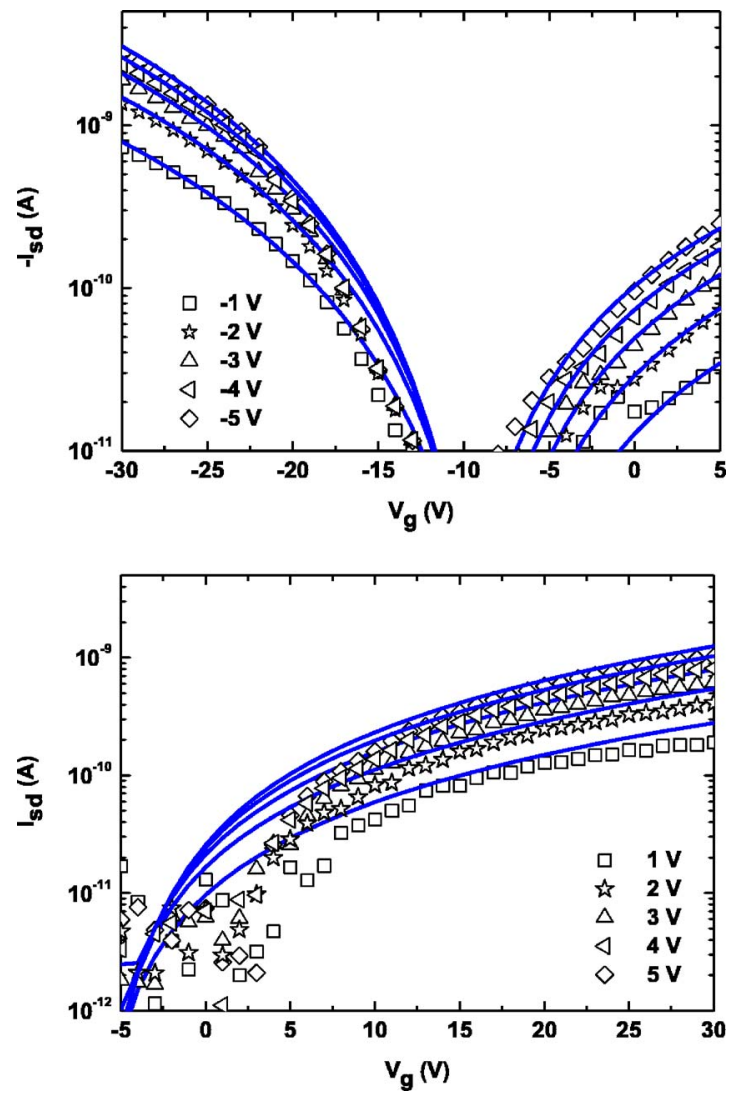

FIG. 4. (Color online) Transfer characteristics of the unipolar regime for negative (a) and positive (b) drain biases varying from $+/-1,2,3,4$, to $5 \mathrm{~V}$. A transistor with a channel length of $40 \mu \mathrm{m}$ was taken and the temperature was fixed at $313 \mathrm{~K}$. The symbols represent the data points and the solid line the theoretical fit. Some contact or subthreshold problems are noticeable for low $V_{d}$ and $V_{g}$.

A set of transfer curves measured in the linear regime at $313 \mathrm{~K}$ is presented in Fig. 4. Symbols are data points and solid lines are theoretical fits. The gate bias was swept back and forth and no hysteresis was observed. Small negative drain biases and negative gate biases shown in Fig. 4(a) correspond to a standard unipolar hole-only transistor in the linear regime (regime 4). Similarly, at low drain biases and positive gate biases, we observe reversed electron-only accumulation (regime 6). In Fig. 4(b), small positive drain biases and positive gate biases correspond to a standard unipolar electron-only transistor in the linear regime (regime 1).

Upon sweeping the gate from positive to negative using a large negative $V_{d}$ [Fig. 5(a)], the operating transport regime changes from reversed electron only (regime 6) to an ambipolar transport regime (regime 5), to hole-only accumulation (regime 4). For a large positive $V_{d}$, the gate can be swept from negative to positive [Fig. 5(b)], where the operating regime goes from reversed hole accumulation (regime 3 ) to ambipolar transport regime (regime 2) to the electron only accumulation (regime 1). All transistors were characterized as a function of temperature between 233 and $313 \mathrm{~K}$. Representative transfer curves are presented in Fig. 6.

One parameter needed for the theoretical description of the current in the ambipolar transistor is the threshold voltage, $V_{t}$. The threshold voltage can be determined indepen-
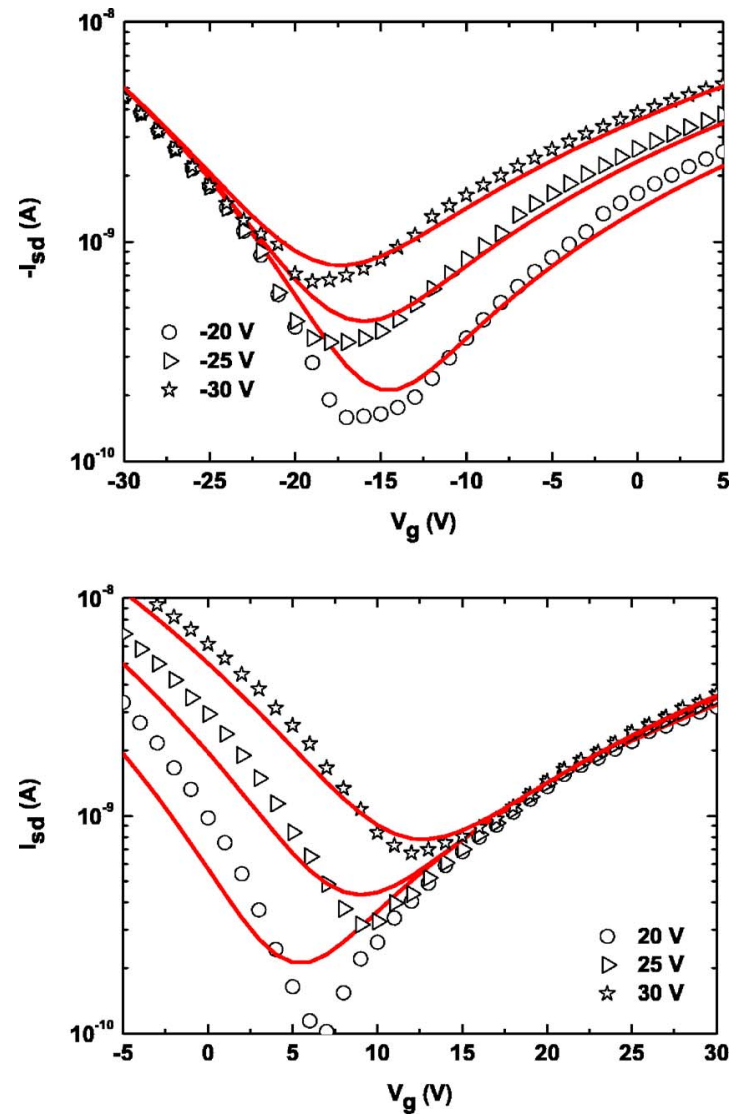

FIG. 5. (Color online) Transfer characteristics of the ambipolar regime for negative (a) and positive (b) drain biases varying from $+/-20,25$, to $30 \mathrm{~V}$. A transistor with a channel length of $40 \mu \mathrm{m}$ was taken and the temperature was fixed at $313 \mathrm{~K}$. The symbols represent the data points and the solid line the theoretical fit.

dently from impedance measurements on MIS diodes, Fig. 1(d). These are a sensitive tool to measure accumulation and depletion of charge carriers as a function of applied bias. We calculated the diode capacitances from the modulus of the impedance, $Z$, and its phase angle, $\Theta$ using $C=$ $-\sin \Theta /(\omega|Z|)$, with $\omega=2 \pi f_{\text {mod }}$, where $f_{\text {mod }}$ is the modulation frequency. In order to minimize dielectric relaxation artefacts, a low modulation frequency of $17 \mathrm{~Hz}$ was chosen. The capacitance of the devices is scaled with the area, which ranges from 0.07 to $0.38 \mathrm{~cm}^{2}$. The capacitance as a function of the bias is presented in Fig. 7. The bias was swept up and down. Only a little hysteresis was observed. Both at high positive and high negative bias, the capacitances are the same and approximately equal to the geometric capacitance of the gate dielectric. This demonstrates that holes are accumulated at high negative bias and electrons at high positive bias. When the gate bias deceases, the semiconductor gets depleted of charge carriers. Therefore, the capacitance decreases with bias and is minimal when the gate bias is equal to the threshold voltage. The threshold voltage is expected to be caused by fixed charges and may therefore be assumed to be the same for both electrons and holes as long as there is no hysteresis. The data confirm the validity of that assumption. The value obtained, $-7 \mathrm{~V}$, can be due to fixed ions or trapped charges at the semiconductor dielectric interface. 

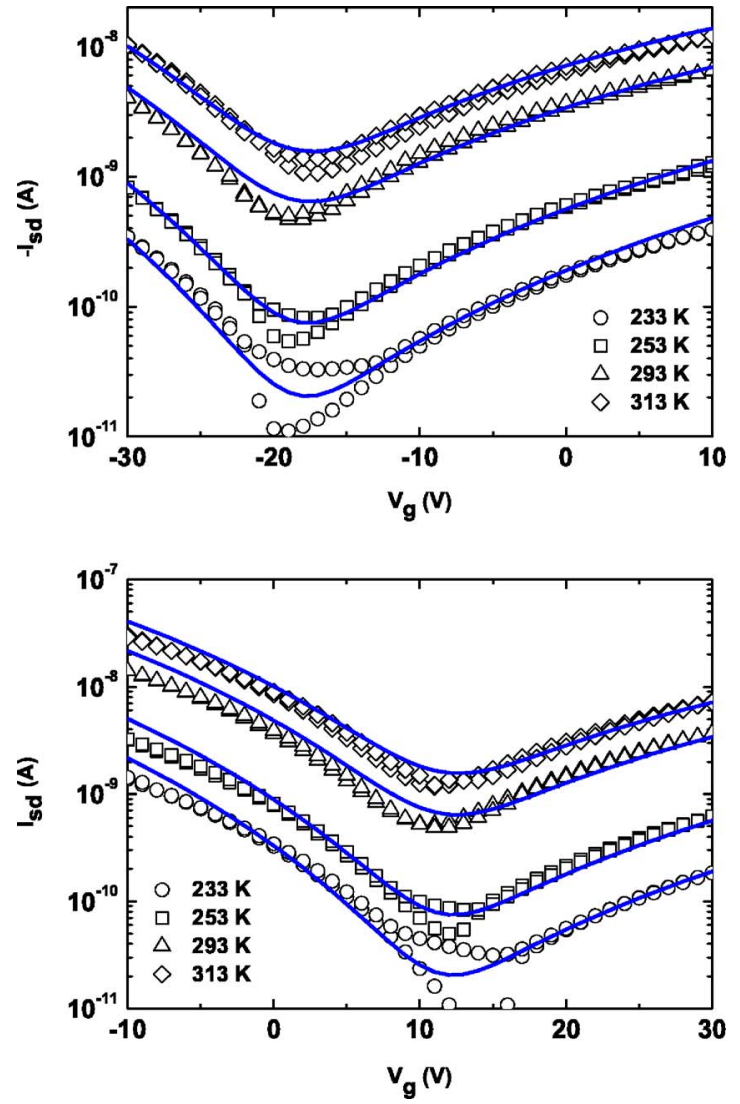

FIG. 6. (Color online) Transfer characteristics for high drain biases with varying temperature from 233 to $313 \mathrm{~K}$. The channel length was $20 \mu \mathrm{m}$ and the drain bias was $-30 \mathrm{~V}$ (a) and $30 \mathrm{~V}$ (b). The symbols represent the data points and the solid line the theoretical fit.

Now that we have determined the threshold voltage, the current transport is given by the following parameters. The temperature describing the width of the exponential density of states, $T_{0}$, a wavelength localization overlap, $\alpha^{-1}$, and the conductivity prefactor, $\alpha_{0}$. These parameters have to be determined for both holes and electrons and were found by

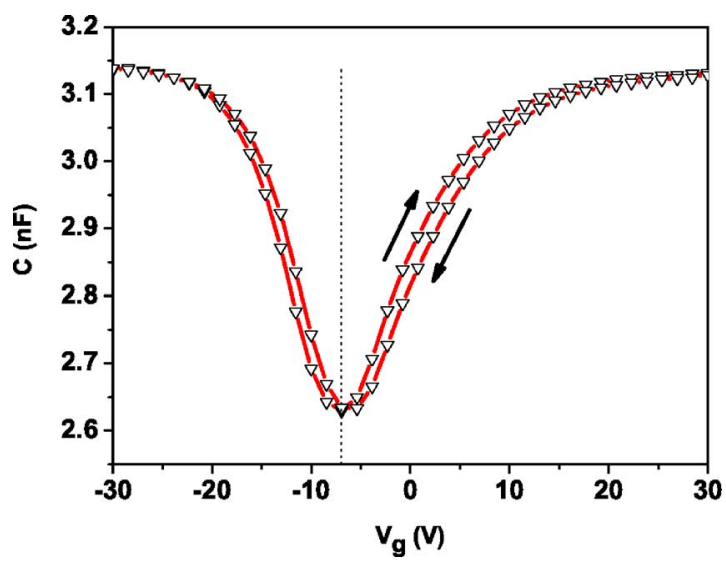

FIG. 7. (Color online) Capacitance of a MIS diode measured at $17 \mathrm{~Hz}$ and $253 \mathrm{~K}$. The hole accumulation at the left and electron accumulation at the right are shown. A minimum in the capacitance is found at $-7 \mathrm{~V}$, which is taken as the threshold voltage.
TABLE I. Values obtained from fitting the transistor data over a wide range of temperatures, drain, and gate biases. The field-effect mobility is given for a temperature of $313 \mathrm{~K}$ and a gate bias +/ $-30 \mathrm{~V}$.

\begin{tabular}{lcccc}
\hline \hline Parameter & $T_{0}(\mathrm{~K})$ & $\sigma_{0}\left(10^{6} \mathrm{~S} / \mathrm{m}\right)$ & $\sigma^{-1}(\AA)$ & $\mu_{\mathrm{FE}}\left[\mathrm{cm}^{2} /(\mathrm{Vs})\right]$ \\
\hline NiDT holes & 600 & 1.9 & 2.1 & $2.5 \times 10^{-4}$ \\
NiDT electrons & 460 & 0.78 & 0.9 & $2.0 \times 10^{-5}$ \\
OC1C10-PPV $^{\mathrm{a}}$ & 540 & 31 & 1.4 & $4.7 \times 10^{-4}$ \\
P3HT $^{\mathrm{a}}$ & 425 & 1.6 & 1.6 & $6.0 \times 10^{-4}$ \\
Pentacene $^{\mathrm{b}}$ & 385 & 3.5 & 3.1 & $2.0 \times 10^{-2}$ \\
PTV $^{\mathrm{b}}$ & 382 & 5.6 & 1.5 & $2.0 \times 10^{-3}$ \\
\hline
\end{tabular}

${ }^{\mathrm{a} R e f e r e n c e} 21$.

${ }^{\mathrm{b}}$ Reference 23 .

fitting the unipolar regimes (regimes 1 and 4) as a function of temperature [Eqs. (9) and (10)]. The fitted curves are presented as solid lines in Fig. 4. With those parameters, the $I(V)$ curves in the ambipolar regimes 2 and 5 were "predicted" (full lines) in Figs. 5 and 6, together with the experimental data [Eq. (18)]. The good agreement between measured and calculated currents demonstrates that our model consistently explains the output and transfer characteristics of these OFETs in the ambipolar regime. Small deviations are noticeable when the gate bias is close to the threshold voltage. This can be due to either contact resistances ${ }^{8}$ or subthreshold current contributions ${ }^{28}$ that are ignored in the present modeling. An underlying assumption is that due to the low mobility, the channel resistance is large compared to the contact resistance. ${ }^{21}$ Values of the fit parameters hardly depend on the channel length that was varied between 20 and $40 \mu \mathrm{m}$. For these channel lengths, we assume that short channel effects can be disregarded in NiDT transistors. Finally, we note that we did not include bulk conductivity of the semiconductor. ${ }^{25}$ The transfer curves show that this contribution can be neglected for the NiDT transistors.

The values of the parameters $\alpha^{-1}, T_{0}$, and $\sigma_{0}$ are presented in Table I. The values for the hole and electron field-effect mobility at $313 \mathrm{~K}$ and $+/-30 \mathrm{~V}$ are included as well. The linear mobility at high bias is close to the saturated mobility. A detailed discussion on the values obtained is beyond the scope of this paper. That would require, for instance, a systematic investigation on the microstructure of the semiconductor and changes therein upon processing. Here we focused on the description of the current-voltage characteristics. However, we note that the values of NiDT in Table I are of a similar order of magnitude for $\alpha^{-1}, T_{0}$, and $\sigma_{0}$ as reported values for, e.g., pentacene, poly(2,5-thienylene vinylene), poly(3-hexyl thiophene), and poly(p-phenylene vinylene) derivatives. ${ }^{20,21}$

\section{CONCLUSION}

A model describing charge transport in disordered ambipolar organic field-effect transistors has been presented. The basis of this model is the variable-range-hopping theory developed for the mobility in disordered unipolar organic tran- 
sistors. An accurate description for the transport as a function of the temperature and gate biasing has been demonstrated for the linear regime. Here, we show that the formalism can be applied to a wide range of bias conditions including saturation and ambipolarity.

First, the charge-carrier density in a sheet as a function of effective gate potential in the channel was solved analytically for an exponential DOS. We substituted the relation between bulk conductivity and charge-carrier density. The sheet conductance followed from integration over the charge-carrier density profile. The source drain current as a function of bias was then obtained by integrating the sheet conductances along the channel. For the special case of an ambipolar transistor, the effective gate potential changes sign in the channel. Holes and electrons are accumulated at opposite sides. A junction separates the accumulation regions, assuming an infinite recombination rate of electrons and holes. The ambipolar transistor is represented by a unipolar $n$-type transistor in series with a unipolar $p$-type transistor. An expression for the position of the $p n$-junction and the current as a function of bias voltages was derived.

The analysis was applied to ambipolar field-effect transistors based on the small band-gap semiconductor nickel dithi- olene. First, the value for the threshold voltage was determined independently from MIS diode measurements. The remaining charge transport parameters, namely the width of the exponential density of states, the wave function overlap localization length, and the conductivity prefactor for holes and electrons, respectively, are subsequently determined from the unipolar response of the transistors. Using these parameters, an excellent agreement between measured and calculated currents is obtained for the ambipolar output and transfer curves as a function of temperature. The parameter values are quite similar to previously reported values for, e.g., pentacene, poly(2,5-thienylene vinylene), poly(3-hexyl thiophene), and poly( $p$-phenylene vinylene) derivatives (see Table I).

\section{ACKNOWLEDGMENTS}

From Philips Research Laboratories, Eindhoven, The Netherlands, we are grateful to Piet Rommers for performing the CV measurements and Henny Herps for his contribution to Fig. 2. We thank Christian Martin for his useful discussions. The work of E.C.P.S. forms part of the research program of the Dutch Polymer Institute (DPI), project no. 516.
*Corresponding author. Email address: e.c.p.smits@rug.nl

${ }^{1}$ N. S. Stingelin-Stutzmann, E. C. P. Smits, H. Wondergem, C. Tanase, P. W. M. Blom, P. Smith, and D. M. De Leeuw, Nat. Mater. 4, 601 (2005).

${ }^{2}$ V. C. Sundar, J. Zaumseil, V. Podzorov, E. Menard, R. L. Willett, T. Someya, M. E. Gershenson, and J. A. Rogers, Science 303, 1644 (2005).

${ }^{3}$ B. Crone, A. Dodabalapur, Y.-Y. Lin, R. W. Filas, Z. Bao, A. LaDuca, R. Sarpeshkar, H. E. Katz, and W. Li, Nature (London) 403, 521 (2000).

${ }^{4}$ B. K. Crone, A. Dodabalapur, R. Sarpeshkar, R. W. Filas, Y.-Y. Lin, Z. Bao, J. H. O’Neill, W. Li, and H. E. Katz, J. Appl. Phys. 89, 5125 (2001).

${ }^{5}$ A. Dodabalapur, H. Katz, L. Torsi, and R. Haddon, Appl. Phys. Lett. 68, 1108 (1996).

${ }^{6}$ E. Kuwahara, Y. Kubozono, T. Hosokawa, T. Nagano, K. Masunari, and A. Fujiwara, Appl. Phys. Lett. 85, 4765 (2004).

${ }^{7}$ Th. B. Singh, F. Meghdadi, S. Günes, N. Marjanovic, G. Horowitz, P. Lang, S. Bauer, and N. S. Sariciftci, Adv. Mater. (Weinheim, Ger.) 17, 2315 (2005).

${ }^{8}$ T. D. Anthopoulos, C. Tanase, S. Setayesh, E. J. Meijer, J. C. Hummelen, P. W. M. Blom, and D. M. De Leeuw, Adv. Mater. (Weinheim, Ger.) 16, 2174 (2004).

${ }^{9}$ T. D. Anthopoulos, D. M. de Leeuw, E. Cantatore, S. Setayesh, E. J. Meijer, C. Tanase, J. C. Hummelen, and P. W. M. Blom, Appl. Phys. Lett. 85, 4205 (2004).

${ }^{10}$ C. Rost, D. Gundlach, S. Karg, and W. Riess, J. Appl. Phys. 95, 5782 (2004).

${ }^{11}$ E. J. Meijer, D. M. De Leeuw, S. Setayesh, E. Van Veenendaal, B.-H. Huisman, P. W. M. Blom, J. C. Hummelen, U. Scherf, and T. M. Klapwijk, Nat. Mater. 2, 678 (2003).

${ }^{12}$ T. D. Anthopoulos, D. M. de Leeuw, E. Cantatore, P. van 't Hof,
J. Alma, and J. C. Hummelen, J. Appl. Phys. 98, 054503 (2005).

${ }^{13}$ M. Shkunov, R. Simms, M. Heeney, S. Tierney, and I. McCulloch, Adv. Mater. (Weinheim, Ger.) 17, 2608 (2005).

${ }^{14}$ T. D. Anthopoulos, E. C. P. Smits, S. Setayesh, M. Coelle, E. Cantatore, and D. M. De Leeuw, Adv. Mater. (Weinheim, Ger.) (to be published).

${ }^{15}$ E. J. Meijer, C. Detcheverry, P. J. Baesjou, E. van Veenendaal, D. M. de Leeuw, and T. M. Klapwijk, J. Appl. Phys. 93, 4831 (2003).

${ }^{16}$ E. J. Meijer, A. V. G. Mangnus, C. M. Hart, D. M. de Leeuw, and T. M. Klapwijk, Appl. Phys. Lett. 78, 3902 (2001).

${ }^{17}$ H. Bässler, Phys. Status Solidi B 175, 15 (1993).

${ }^{18}$ A. Dieckmann, H. Bassler, and P. M. Borsenberger, J. Chem. Phys. 99, 8116 (1993).

${ }^{19}$ M. C. J. M. Vissenberg and M. Matters, Phys. Rev. B 57, 12964 (1998).

${ }^{20}$ E. J. Meijer, C. Tanase, P. W. M. Blom, E. van Veenendaal, B.-H. Huisman, D. M. de Leeuw, and T. M. Klapwijk, Appl. Phys. Lett. 80, 3838 (2002).

${ }^{21}$ C. Tanase, E. J. Meijer, P. Blom, and D. de Leeuw, Org. Electron. 4, 33 (2003).

${ }^{22}$ M. Shur, M. Hack, and J. G. Shaw, J. Appl. Phys. 55, 3831 (1984).

${ }^{23}$ S. Kishida, Y. Naruke, Y. U., and M. Matsumura, Jpn. J. Appl. Phys. 22, 511 (1983).

${ }^{24}$ W. F. Pasveer, J. Cottaar, C. Tanase, R. Coehoorn, P. A. Bobbert, P. W. M. Blom, D. M. de Leeuw, and M. A. J. Michels, Phys. Rev. Lett. 94, 206601 (2005).

${ }^{25}$ E. Calvetti, L. Colalongo, and Zs. M. Kovács-Vajna, Solid-State Electron. 49, 567 (2005).

${ }^{26}$ G. Horowitz, R. Hajlaoui, and P. Delannoy, J. Phys. III 5, 355 (1995). See Appendix A. 
${ }^{27}$ A. Brown, C. Jarrett, D. de Leeuw, and M. Matters, Synth. Met. 88, 37 (1997).

${ }^{28}$ S. F. Nelson, Y.-Y. Lin, D. J. Gundlach, and T. N. Jackson, Appl. Phys. Lett. 72, 1854 (1998).

${ }^{29}$ S. M. Sze, Physics of Semiconductor Devices (WileyInterscience, New York 1981).

${ }^{30}$ R. Schmechel, M. Ahles, and H. von Seggern, J. Appl. Phys. 98, 084511 (2005).

${ }^{31}$ G. Gelinck, E. Van Veenendaal, and R. Coehoorn, Appl. Phys.
Lett. 87, 073508 (2005).

${ }^{32}$ H. Klauk, Organic Electronics (Wiley-VCH, Weinheim, 2006).

${ }^{33}$ G. W. Neudeck, K. Y. Chung, and H. Bare, IEEE Trans. Electron Devices 34, 9383 (1987).

${ }^{34}$ G. Paasch, Th. Lindner, C. Rost-Bietsch, S. Karg, W. Riess, and S. Scheinert, J. Appl. Phys. 98, 084505 (2005).

${ }^{35}$ L. L. Chua, J. Zaumseil, J. F. Chang, E. C. Ou, P. K. Ho, H. Sirringhaus, and R. H. Friend, Nature (London) 434, 194 (2005). 